\title{
Utilidades del certificado digital Izenpe en las bibliotecas públicas de Euskadi
}

\author{
Por Francisca Pulgar Vernalte y Sonia Marcos Maciá
}

\begin{abstract}
Resumen: Este artículo describe algunos de los usos del certificado digital (Izenpe) en el ámbito de los servicios bibliotecarios de Euskadi, y en concreto su utilización para el trabajo en red de los profesionales, mediante el cual acceden al servidor único donde se almacenan los datos, tanto bibliográficos como de lectores. Asimismo se describe el uso del certificado digital como carnet único para el acceso de los socios a los servicios de préstamo, internet, etc., que se ofrecen desde las distintas bibliotecas municipales que integran la red en línea del Sistema Nacional de Bibliotecas de Euskadi (SNBE).
\end{abstract}

Palabras clave: Red de bibliotecas, Carnet único, Servicios en línea, Certificado digital, E-administración.

Title: Izenpe digital signature utilities in public libraries of the Basque country

Abstract: This article describes the uses of digital signatures within Euskadi (Basque country) library services. In particular it focuses on their use for the librarians' networked activities, in which they can access the single server where both bibliographic and user data are stored. Additionally, mention is made of the use of a digital certificate as a single membership card allowing members to access the services (such as circulation, internet, etc.) that are offered by municipal libraries making up the online network of the National System of Libraries of Euskadi.

Keywords: Library network, Membership card, On-line services, D-signature, E-administration.

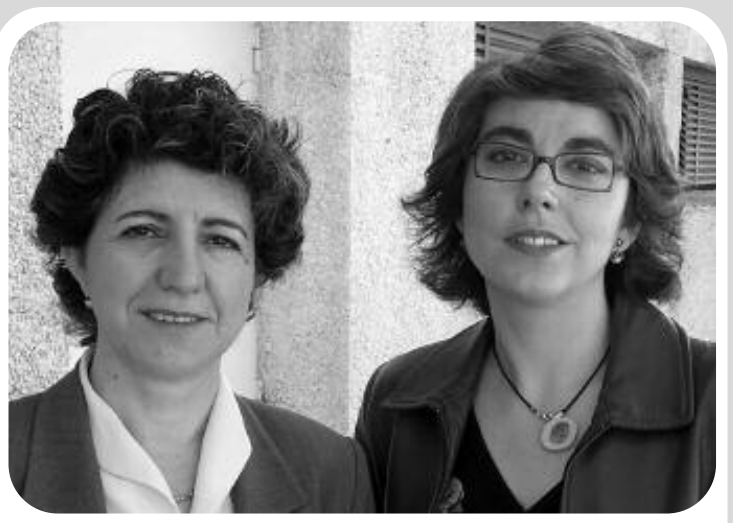

Francisca Pulgar Vernalte, licenciada en filología hispánica por la Universidad de Sevilla. Desde 1983 a 1985 trabajó como becaria en la biblioteca de la Facultad de Geografía e Historia de Vitoria-Gasteiz. En 1985 ocupa el puesto de bibliotecaria en la biblioteca del Departamento de Hacienda del Gobierno Vasco. Desde el año 2000 es responsable del Servicio de Bibliotecas del Gobierno Vasco, gestionando la red de bibliotecas públicas que integran el Sistema Nacional de Bibliotecas de Euskadi.

Sonia Marcos Maciá, licenciada en geografía e historia por la Universidad de Deusto. Desde 1997 ha realizado labores de bibliotecaria y documentalista en diversos centros tanto públicos como privados. En 2003 se incorpora al proyecto de la puesta en marcha de la red en línea del Sistema $\mathrm{Na}^{-}$ cional de Bibliotecas de Euskadi en el que lleva a cabo tareas de coordinación.

Pulgar Vernalte, Francisca; Marcos Maciá, Sonia. «Utilidades del certificado digital Izenpe en las bibliotecas públicas de Euskadi». En: El profesional de la información, 2006, julio-agosto, v. 15, n. 4, pp. 307-309.

\section{Introducción}

EN 2004 SE PUSO EN MARCHA el proyecto de la red en línea de las bibliotecas públicas de Euskadi con el que además de ofrecer un catálogo colectivo consultable por internet se pretendía potenciar el uso y la oferta de los servicios bibliotecarios entre la población de la Comunidad Autónoma de Euskadi (CAE).

Para ello, uno de los primeros pasos fue la selección de un programa de gestión bibliotecaria que permitiera el trabajo en línea y al que se accediera vía web desde los distintos municipios. Tras valorar varios de ellos, se seleccionó absys$N E T$, que fue instalado en un servi- dor de EJIE (Sociedad informática del Gobierno Vasco) con el fin de gestionar los datos bibliográficos que conforman el catálogo en línea de las 219 bibliotecas municipales integradas. Además, a través de este servidor se accede a la base única de lectores.

Se requería definir el nuevo método de trabajo de los 500 bibliotecarios al servidor de EJIE de forma que se garantizara la seguridad que exige este tipo de procesos.

Por otro lado, la implantación del carnet único como una de las ventajas que ofrecía la red a todos los socios de las bibliotecas, planteaba la necesidad de cambiar el que se venía utilizando en cada centro.
De ahí que se considerara que el certificado digital podría ser una buena solución para dar respuesta a los dos requisitos básicos de la red: el acceso al servidor de los bibliotecarios municipales para el trabajo cooperativo por un lado, y la obtención de un carnet único de lector para la utilización de los servicios bibliotecarios tanto en línea como presenciales que se ofrecen a la ciudadanía, por otro. El único requisito legal que se exige es haber cumplido los 16 años.

Este certificado es emitido por Izenpe, una entidad certificadora creada entre el Gobierno Vasco y las diputaciones forales de los tres 
territorios históricos de la CAE (Álava, Bizkaia y Gipuzkoa). http://www.izenpe.com

\section{Conexión de los bibliotecarios al servidor}

Desde el inicio del proyecto se implantó el certificado digital como garantía de acceso de los profesionales al programa de gestión bibliotecaria. Puesto que su utilización es obligatoria, el primer paso que se debe realizar es su obtención (figura 1).

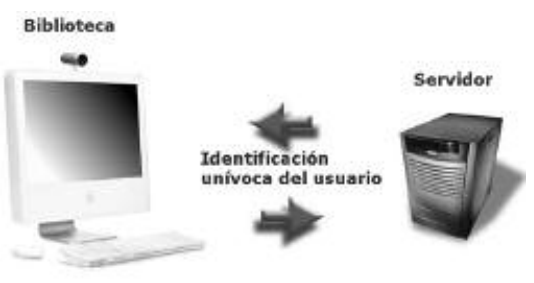

Figura 1. Esquema de conexión del usuario bibliotecario al programa

\subsection{Certificado Izenpe}

Es un identificador único que avala la autenticidad de las operaciones y las transacciones electrónicas, en nuestro caso el trabajo con el programa de gestión bibliotecaria (figura 2).

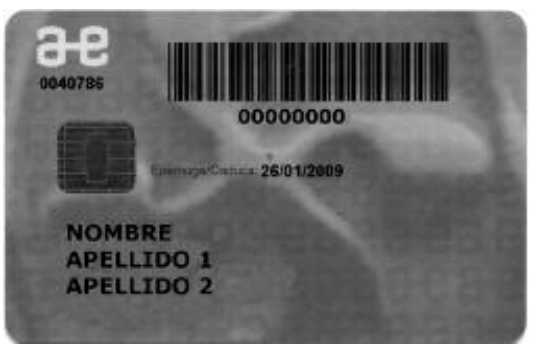

Figura 2. Tarjeta Izenpe

\subsubsection{Tipos de certificados}

-De personal de entidades públicas. Se expide a profesionales con un vínculo laboral directo con el ayuntamiento, ya sea funcionario, interino o laboral temporal.

-De ciudadano. Lo recibe el personal que, aun trabajando para la biblioteca, esté contratado a través de un tercero (empresa).

\subsubsection{Obtención}

Para conseguirlo existen dos procedimientos dependiendo del tipo:

-El profesional de la biblioteca rellena un impreso y lo entrega al secretario/a del ayuntamiento. Éste lo tramita, enviándolo a Izenpe por correo ordinario.

- Los ciudadanos conectan a la web de Izenpe y llaman por teléfono para concertar cita y recoger la tarjeta.

«El nuevo
método de
trabajo en red
requería definir
el acceso de los
bibliotecarios al
servidor, de
forma que se
garantizara la
seguridad que
exige este tipo
de procesos»

En ambos casos Izenpe entrega el certificado digital, junto con un número pin y otro puk, así como un dispositivo lector y un software de instalación para su utilización desde cualquier PC. Todo el proceso sigue un estricto protocolo de seguridad que cumple las normas relativas a la firma digital.

\subsection{Conexión a la aplicación} de gestión bibliotecaria

El usuario podrá acceder al programa de gestión bibliotecaria siguiendo los siguientes pasos:

a. Introducir la tarjeta en el lector (conectado al PC a través del puerto $U S B)$.

b. Conectar con la dirección web de la aplicación. Aparecerá un cuadro de diálogo con el nombre del usuario de la tarjeta. c. En otro cuadro se debe teclear el número pin.

d. Se abrirá el programa y se podrá empezar a trabajar.

\section{Acceso a los servicios bibliotecarios}

El carnet de lector es el documento que acredita como socio de la biblioteca y permite acceder a servicios tales como préstamo de material bibliográfico, la conexión a internet, etc. Hasta la puesta en marcha de la red en línea, cada biblioteca emitía el suyo propio, con lo que si cualquier persona quería o necesitaba acudir a las bibliotecas de otros municipios debía hacerse con uno para cada una de ellas.

El certificado se puso en marcha en marzo de 2005, tras haberse realizado un estudio piloto en Arrasate-Mondragón (Guipúzcoa), Llodio (Álava) y Leioa (Bizkaia). Valorados los resultados se fue ampliando paulatinamente la emisión del certificado digital al resto.

$$
\begin{gathered}
\text { «La } \\
\text { identificación de } \\
\text { usuario y } \\
\text { contraseña se ha } \\
\text { sustituido por } \\
\text { una conexión } \\
\text { usando el } \\
\text { certificado digital } \\
\text { Izenpe» }
\end{gathered}
$$

A principios de 2006 son cerca de 30 los centros que se han apuntado a esta iniciativa, habiéndose repartido unas 6.700 tarjetas (casi el $20 \%$ del total de carnets emitidos desde dicha fecha). Se están incorporando Basauri, Vitoria-Gasteiz, Galdakao, Irún, etc.

\subsection{Servicios bibliotecarios} en línea

Al igual que está ocurriendo con la e-administración, que fomenta la conexión y las relaciones 
entre las administraciones y los ciudadanos vía web, las bibliotecas vascas ofrecen a sus socios la posibilidad de acceder online a determinados servicios bibliotecarios con la opción "Mi biblioteca". Desde cualquier lugar pueden consultar al opac de las bibliotecas participantes en el catálogo en línea del Sistema Nacional de Bibliotecas de Euskadi $(S N B E)$, con una interfaz única. http://www.liburutegiak.euskadi.ne t/katalogobateratua

Si el usuario que se conecta es poseedor de un certificado sólo tiene que pinchar sobre la imagen de la tarjeta en la página principal del opac e introducir el número pin (figura 3).

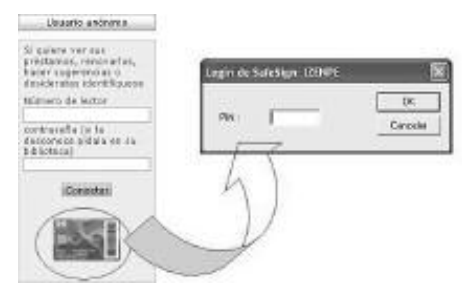

Figura 3. Esquema de identificación del usuario con Izenpe en el opac

Una vez identificado podrá acceder a:

a. Búsqueda y recuperación de información bibliográfica. Consulta sobre los fondos de las 219 bibliotecas integradas en la red.

b. Estado y renovación de los préstamos.

c. Realización y anulación de peticiones de préstamos y reservas.

d. Envío de desideratas.

e. Elaboración de bibliografías personalizadas. Pueden visualizarse, guardarse en un fichero o enviar por e-mail.

f. Acceso a guías de lectura de temas de interés preparadas por profesionales.

g. Posibilidad de comunicar opiniones, sugerencias, etc., a las bibliotecas.

h. Acceso gratuito a recursos electrónicos (Ocenet Saber, Harluxet y la hemeroteca Iconoce).

\section{Otras utilidades}

Se continúa trabajando en la implementación de nuevos servicios usando el certificado digital. Un aspecto positivo de este proceso es que se emite ya con el código de barras necesario para los servicios de préstamo y devolución de materiales en las bibliotecas públicas.

En esta línea es interesante comentar que en la biblioteca de Abadiño (Bizkaia) se utiliza tanto para acceder a los servicios de préstamo y devolución de materiales bibliográficos, como para supervisar el servicio de mediateca. De este modo se controlan las sesiones de acceso a internet de cada usuario.

«Con la misma
tarjeta con la que
se accede a los
servicios
administrativos,
cualquier
ciudadano puede
gestionar
renovaciones,
tramitar
peticiones de
libros, anular
reservas, etc.»

En Basauri, uno de los municipios más populosos de Bizkaia (44.052 habitantes), el certificado digital expedido en la biblioteca también se va a extender a otros servicios municipales como la consulta de datos fiscales, trámites administrativos, acceso a las instalaciones deportivas municipales, etc.

En breve se utilizará el certificado digital para identificar a los usuarios de Kzgunea, una red de centros públicos gratuitos para la formación y el uso de las nuevas tecnologías de la información y comunicación puesto en marcha en 2001

También es utilizado en varios municipios de Gipuzkoa para gestión de certificados de padrón, tasas, impuestos, recibos y multas. Además, entidades como la Agencia Tributaria Estatal (AEAT), la Seguridad Social y la Fundación Tripartita han homologado su utilización.

\section{Bibliografía}

Gutiérrez Lizarralde, Begoña. "E-administración vasca: conjugando planos y ejes arquitectónicos". En: VIII Jornadas sobre tecnologías de la información para la modernización de las administraciones públicas, Tecnimap, 2004. Consultado en: 10-03-06.

http://www.csi.map.es/csi/tecnimap/tecnimap_20 04/comunicaciones/tema_06/6_011.pdf

Instituto Nacional de Estadística. Consultado en: 10-03-06.

http://www.ine.es

Kzgunea. Consultado en: 10-03-06. http://www.kzgunea.net

Pulgar Vernalte, Francisca; Marcos Maciá, Sonia. «La red en línea de las bibliotecas públicas de Euskadi: un instrumento para la cooperación». En: II Jornadas bibliotecarias de Castilla-La Mancha, 2005. Consultado en:

10-03-06.

http://www.kultura.ejgv.euskadi.net/r46-4879/es /contenidos/informacion/actas_y_informes/es_9 306/adjuntos/es_Jor_Bib_Toledo.pdf

Sánchez Martínez, Daniel; Cascales Saseta, José Antonio; Ferrando Martínez, Rafael; Pérez Guzmán, Francisco José. «E-administración en la universidad de Murcia». En: VIII Jornadas sobre tecnologías de la información para la modernización de las administraciones públicas, Tecnimap, 2004. Consultado en: 10-03-06.

http://www.csi.map.es/csi/tecnimap/tecnimap_20 04/comunicaciones/tema_06/6_025.pdf

Francisca Pulgar Vernalte, Servicio de Bibliotecas, Dpto. de Cultura, Gobierno Vasco.

C/ Donostia-San Sebastián 1, 01010 Vitoria-Gasteiz.

Tel.: +34-945019474

f-pulgar@ej-gv.es

Sonia Marcos Maciá, ODEI SA, Dpto. de Cultura, Gobierno Vasco.

C/ Donostia-San Sebastián 1, 01010 Vitoria-Gasteiz.

Tel.: +34-945019474

smmacia@gmail.com 